\title{
Initiative
}

\section{AOK fördert die Nachsorge von schwerkranken Kindern}

\begin{abstract}
Kinder, die an schweren Krankheiten leiden, sollen so wenig Zeit wie möglich im Krankenhaus verbringen. Um dieses Ziel zu erreichen, muss die ambulante Versorgung von Kindern in Deutschland ausgebaut werden. Die AOK hat einen ersten wichtigen Schritt unternommen.
\end{abstract}

- Sozialmedizinische Nachsorge wird seit 2004 in $\S 43$ Abs. 2 SGB V geregelt, doch bislang wurde noch kein einziger Vertrag auf dieser Grundlage geschlossen. Speziellen Bedarf haben Familien mit schwerkranken Kindern beim Übergang von der Klinik nach Hause.

Den bisher ersten Vertrag zur Nachsorge für Kinder unter zwölf Jahren hat die AOK Baden-Württemberg in Heilbronn mit dem „Bunten Kreis“ abgeschlossen. Die Kasse übernimmt künftig die Kosten für eine ausgebildete Nachsorgeschwester, die die Familie in der besonders schwierigen Übergangsphase nach der Klinikentlassung unterstützt.

Die Nachsorge darf nur von Einrichtungen ausgeführt werden, die die Qualitätsanforderungen der AOK erfüllen, erklärte Dr. Christopher Hermann, stellvertretender Vorsitzender der AOK Ba-
den-Württemberg, bei der Vertragsunterzeichnung in Heilbronn. „Diesen Anforderungen entspricht der ,Bunte Kreis' Heilbronn, mit dem wir deshalb jetzt den ersten Vertrag dieser Art unterzeichnen."

Der „Bunte Kreis“ ist 1998 in Augsburg entstanden. Inzwischen gibt es 20 solcher Einrichtungen in Deutschland. Sie werden weitgehend von Spendenund Sponsorengeldern finanziert, der Hauptförderer ist die Stiftung des Augsburger Generikaherstellers betapharm.

Die AOK erhofft sich von dem Vertrag sowohl eine bessere Nachsorge der Kinder und ihrer Familien als auch finanzielle Einsparungen durch Vermeidung bzw. Verkürzung stationärer Aufenthalte. Eine Studie hat gezeigt, dass beispielsweise durch die Nachsorge von frühgeborenen Kindern pro Kind zwi-

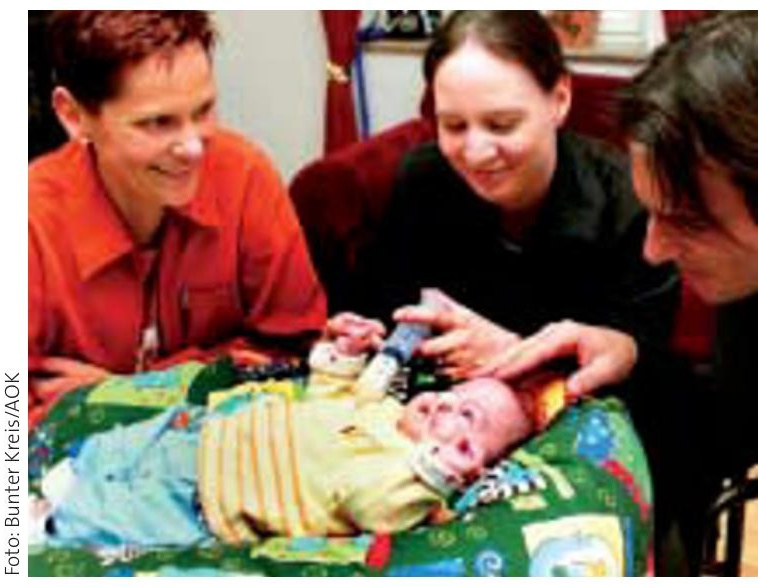

Besonders Eltern von schwerkranken Säuglingen profitieren von der Nachsorge.

schen 1500 und 3000 Euro jährlich eingespart werden können.

Die Nachsorgeschwestern bieten Trost, Beratung und Hilfe bei der aufwendigen Pflege. Sie helfen aber auch beim Antrag auf einen Schwerbehindertenausweis oder eine Haushaltshilfe oder bei der Pflegeeinstufung des Kindes. In finanziellen Notsituationen steht ein Sozialfonds zur Verfügung. Ganz wichtig ist dem „Bunten Kreis“ die Betreuung von Familien in der schwersten Krisensituation: dann, wenn ein Kind sterben muss. Auch diese Leistung wird von der AOK mitgetragen, betonte Christopher Hermann in Heilbronn.

KS

\section{Aktuelle Abrechnungstipps}

\section{Komplexziffer mit großem Potenzial}

Wie kann ich mit dem EBM heute noch Geld verdienen? Gibt es Abrechungstricks? Dr. Roman $\mathrm{Ma-}$ chens, Allgemeinarzt in Landshut, kennt sich in diesem schwierigen Terrain aus. Hier einige Tipps.

Wirkliche Tricks gibt es nicht, aber gute und weniger gute Abrechnung gibt es durchaus! Beachten Sie zunächst die juristischen „Basics“:

- Alles abrechnen, was Sie tun!

- Keinesfalls nicht erbrachte Leistungen abrechnen!

- Erbringen Sie die aus Ihrer Sicht medizinisch notwendige Leistung, auch wenn Sie sie nicht abrechnen können - aber sagen Sie das auch dem Patienten!

Bei Komplexziffern sollten Sie aus betriebswirtschaftlicher Sicht nur den vorgeschriebenen, obligaten Teil erbringen und den fakultativen Teil weglassen - natürlich nur soweit dies aus medizinischer Sicht verantwortbar ist. Bei Patienten mit chronisch internistischen Erkrankungen lohnt es sich, die mit 455 Punkten hoch bewertete EDVNummer „03210“ einzusetzen. Stellen Sie die Diagnose „chronisch internistisch" so häufig, wie es sich unter den Kollegen eingebürgert hat, nämlich in vielen $\mathrm{KV}$-Bereichen bei $80 \%$ der
Hausarztpatienten. Sie finden den durchschnittlichen Prozentsatz für Ihre KV-Region übrigens in Ihrer KV-Abrechnung in der Spalte mit dem Vergleich Ihrer Praxis mit der Fallgruppe. Halten Sie sich annähernd an diesen Durchschnittssatz, dann werden Sie nicht auffällig und erreichen das Abrechnungsoptimum.

Doch Vorsicht: Wenn Sie bei diesen Patienten im selben Quartal noch ein EKG mit 03320 oder eine Lungenfunktion mit 03330 abrechnen würden, streicht die KV diese automatisch wieder heraus, denn sie sind fakultative Leistungsbestandteile der Betreuungsziffer 03210! 